\title{
3,5,4'-Tri-O-acetylresveratrol attenuates seawater inhalation-induced acute respiratory distress syndrome via thioredoxin 1 pathway
}

\author{
YILIN ZHAO ${ }^{1 *}$, LIJIE MA ${ }^{1,2^{*}}$, RUIXUAN WANG $^{1}$, TINGTING CHEN $^{4}$, XUEYING LIU $^{3}$ and FAGUANG JIN ${ }^{1}$ \\ ${ }^{1}$ Department of Respiration, Tangdu Hospital, The Fourth Military Medical University, Xi'an, Shaanxi 710038; \\ ${ }^{2}$ Department of Respiration, Chengdu Military General Hospital, Chengdu, Sichuan 610083; \\ ${ }^{3}$ Department of Medicinal Chemistry, School of Pharmacy, The Fourth Military Medical University; \\ ${ }^{4}$ School of Accounting, Xijing University, Xi'an, Shaanxi 710032, P.R. China
}

Received September 11, 2015; Accepted January 12, 2018

DOI: $10.3892 / \mathrm{ijmm} .2018 .3528$

\begin{abstract}
The protecting effects of 3,5,4'-tri-O-acetylresveratrol (AC-Res) on seawater inhalation-induced acute respiratory distress syndrome (ARDS) by interfering with the activation of thioredoxin-1 (Trx-1) pathway were evaluated. Seawater inhalation-induced ARDS was assessed by magnitude of pulmonary edema and lung inflammation. Oxidative stress was tested by T-SOD activity and MDA content in lungs and cells. Besides, Trx-1, nuclear factor erythroid 2-related factor 2 (Nrf2) and Txnip expression were measured to explore how seawater induced oxidative stress and the mechanism by which AC-Res attenuated seawater inhalation-induced ARDS. The results showed that seawater inhalation increased wetto-dry (W/D) ratios of lung tissues, enhanced secretion of tumor necrosis factor- $\alpha$ (TNF- $\alpha)$ and interleukin-1 $\beta$ (IL-1 $\beta$ ), and disturbed the oxidative distress balance probably through interfering the activity of Trx-1 pathway. While treatment of AC-Res in vivo and Res in vitro reduced W/D ratios of lung tissues, decreased cytokines in lungs and maintained the oxidative stress balance through Trx-1 pathway. In conclusion, AC-Res treatment attenuated seawater inhalation induced ARDS via Trx-1 pathway.
\end{abstract}

Correspondence to: Professor Faguang Jin, Department of Respiration, Tangdu Hospital, The Fourth Military Medical University, Xi'an, Shaanxi 710038, P.R. China

E-mail: jinfag@fmmu.edu.cn

Professor Xueying Liu, Department of Medicinal Chemistry, School of Pharmacy, The Fourth Military Medical University, Xi'an, Shaanxi 710032, P.R. China

E-mail: weiyingxuan427@163.com

${ }^{*}$ Contributed equally

Key words: 3,5,4'-tri-O-acetylresveratrol, seawater, acute respiratory distress syndrome, thioredoxin 1 pathway

\section{Introduction}

Acute respiratory distress syndrome (ARDS) is a common and devastating complication which is usually caused by systemic inflammatory response syndrome (SIRS), severe trauma and direct lung injury resulted from inhalation of toxic substances, such as toxic gases and fluids (1). Among various kinds of accidental aspiration, drowning has been recognized as a serious public health problem, which was also the third most frequent causes for accidental death and led to $\sim 359,449$ deaths in 2011 according to the latest statistical data from the world health organization (WHO) (2). Except for sudden death on the spot where drowning happens, injuries and deaths following near-drowning depend on both the composition and quantity of water aspirated (3), as well as the quality of following treatment (4). Compared with the injuries caused by fresh water, previous studies have indicated that seawater inhalation results in more infiltration of inflammatory cells, such as neutrophils, monocytes and lymphocytes, and more secretion of pro-inflammatory mediators including $\mathrm{NO}, \mathrm{COX}-2$, tumor necrosis factor- $\alpha$ (TNF- $\alpha$ ), interleukin-1 $\beta$ (IL-1 $\beta$ ) and IL-6 (5). Besides, it has also been proven that hypertonic stimulation as well as the concentration of inflammatory cells could lead to excessive generation of reactive oxygen species (ROS) (6), however, there is little research showing the role of oxidative stress in seawater inhalation-induced ARDS.

The excessive generation of ROS is the primary cause for oxidative stress imbalance which is closely related to the concentration of inflammatory cells and secretion of cytokines $(7,8)$. As one of the crucial aspects for lung injuries, the generation of ROS is counterbalanced by antioxidant systems. Among which, thioredoxin (Trx) system, composed of NADPH, thioredoxin reductase (TrxR), Trx-1 and Prdxs, is one of the most important systems. It is well known that super-abundant ROS can evoke a series of antioxidant genes by activating nuclear factor erythroid 2-related factor 2 (Nrf2), which is a critical transcription factor regulating the expression of major antioxidant enzymes and phase II detoxification enzymes (9). There is also evidence indicating that the expression of Nrf2 would subsequently induce Trx-1 expression (10). While as 
negative regulator, thioredoxin-interacting protein (Txnip) binds to Trx-1 and suppresses Trx-1 mediated pro-survival signaling and antioxidant process (11). Up to now, there is little evidence revealing the regulation of $\operatorname{Tr} x-1$ axis on hypertonic stimulation induced ROS and there are also limited material showing how Trx-1-related pathway participated in seawater inhalation induced ARDS.

Resveratrol is a natural compound which can be found in grapes, nuts and red wine, it is also one of most intensively researched natural products for its multiple protecting effects. Evidence show that resveratrol (Res) possesses anti-inflammation property probably through interfering the activity of sirtuin 1 (SIRT1) and following inflammatory regulators $(12,13)$. There are also studies indicating that Res exhibited its anti-oxidative stress ability via varied pathways (14). Although Res possesses multiple biological and pharmacological activities, it could not be adopted as a drug in clinic for its poor pharmacokinetic and bio-availability properties (15). While as a prodrug of Res, 3,5,4'-tri-O-acetylresveratrol (AC-Res) prolongs the half-time of Res and caused the accumulation of Res in lungs (16). Studies from our laboratory and other teams have also revealed the biological benefits of AC-Res (17); however, investigations are still needed to further illustrate the pharmacological activity of AC-Res.

Based on what is known, we hypothesized and investigated, in the present study, seawater inhalation-caused inflammation and oxidative stress in lungs, and abnormal expression of Trx-1 pathway was found to cause the process. While administration of AC-Res could protect lungs against seawater exposureinduced ARDS by regulation the expression of Trx-1 axis and following oxidative stress.

\section{Materials and methods}

Animals and reagents. Adult male Sprague-Dawley (SD) rats weighing 200-220 g were provided by the Animal center of the Fourth Military Medical University (FMMU, Xi'an, China). Rats were captured in air-filtered, temperature-controlled units with free access to food and water. All experimental protocols were approved by the Animal Care and Use Committee of the FMMU according to the Declaration of the National Institutes of Health Guide for Care and Use of Laboratory Animals (publication no. 85-23, revised 1985).

Seawater was prepared according to the formula provided by Chinese Ocean Bureau: osmolality 1,300 $\mathrm{mOsm} / 1$, $\mathrm{pH}$ 8.2, $\mathrm{SW} 1.05, \mathrm{NaCl}_{2} 6.518 \mathrm{~g} / 1, \mathrm{MgSO}_{4} 3.305 \mathrm{~g} / 1, \mathrm{MgCl}_{2}$ $2.447 \mathrm{~g} / 1, \mathrm{CaCl}_{2} 1.141 \mathrm{~g} / 1, \mathrm{KCl} 0.725 \mathrm{~g} / 1, \mathrm{NaHCO}_{3} 0.202 \mathrm{~g} / 1$, $\mathrm{NaBr} 0.083 \mathrm{~g} / \mathrm{l}$. Artificial seawater was sterilized before experiments. Resveratrol (3,5,4'-trihydroxystilbene, Res, structure is shown in Fig. 1A), was purchased from Xi'an Grass Plant Technology Corp. (Xi'an, China) with purity $>98 \%$. AC-Res, structure (Fig. 1B), was synthesized by the Pharmacy Department of Medicinal Chemistry of FMMU with HPLC purity $>99 \%$. Enzyme-linked immunosorbent assay (ELISA) kits for TNF- $\alpha$ and IL-1 $\beta$ were purchased from the R\&D Systems Inc. (Minneapolis, MN, USA). MDA and T-SOD activity analyzing kits were purchased from Jiancheng Bioengineering Institute (Nanjing, China). Antibodies against Nrf2, Trx-1, Txnip and $\beta$-actin were purchased from the Santa Cruz Biotechnology, Inc. (Santa Cruz, CA, USA).
Modeling and grouping. SD rats were randomly assigned into 4 groups $(\mathrm{n}=8)$ : control $(\mathrm{Ctl})$ group; seawater $(\mathrm{SW})$ inhalation group; SW + AC-Res group; AC-Res group. Rats from $\mathrm{SW}+\mathrm{AC}-\mathrm{Res}$ group and AC-Res group were pretreated with AC-Res (50 mg/kg/day) for 7 days, and rats from $\mathrm{Ctl}$ and SW group were treated with equal amount of normal saline. Seawater was instilled into the trachea of rats from SW and SW + AC-Res group 90 min after the last administration of normal saline or AC-Res.

The seawater inhalation rat models were built according to a previous study in our laboratory. Briefly, the trachea was exposed after the rat was anesthetized with $3 \%$ pentobarbital sodium $(100 \mathrm{mg} / \mathrm{kg}$ body weight, i.p.). A $1 \mathrm{ml}$ syringe was gently stabbed into the trachea and $4 \mathrm{ml} / \mathrm{kg}$ body weight of artificial seawater was instilled into both lungs within $4 \mathrm{~min}$. Rats were held in the supine position with the head elevated $30^{\circ}$ degree and maintained anesthesia with $25 \mathrm{mg} / \mathrm{kg} / \mathrm{h}$ pentobarbital sodium. Rats from all groups were sacrificed $4 \mathrm{~h}$ after modeling.

Cell culture and treatment. The alveolar macrophage cell line NR8383 and alveolar epithelial cell line A549 were cultured to evaluate the protecting effects of Res, intermediate of AC-Res. NR8383 cells were maintained in Ham's F12 and A549 cells in RPMI-1640 medium containing $10 \%$ fetal calf serum at $37^{\circ} \mathrm{C}$ in a humidified atmosphere containing $5 \% \mathrm{CO}_{2}$ and $95 \%$ air. Cells within the logarithmic growth phase were divided into four groups: control $(\mathrm{Ctl}) ; 25 \%$ seawater treated only (SW); $25 \%$ seawater $+40 \mu \mathrm{g} / \mathrm{ml}$ resveratrol $(\mathrm{SW}+\mathrm{Res}) ; 40 \mu \mathrm{g} / \mathrm{ml}$ Res. Cells from SW and SW + Res group were stimulated with $25 \%$ seawater with the presence of Res or not. All cells were collected after being treated for $4 \mathrm{~h}$.

Lung wet-to-dry (W/D) weight ratios. In order to quantify the magnitude of pulmonary edema, W/D weight ratio of lung samples were calculated. Briefly, lung tissues of the same lob from each rat in every group were obtained and weighed immediately. After that, lung samples were kept in an oven at $70^{\circ} \mathrm{C}$ for $72 \mathrm{~h}$. Finally, samples were weighed again and W/D was calculated by dividing the wet weight with the dry.

Analysis of cytokines. Contents of TNF- $\alpha$ and IL-1 $\beta$ in lung tissues and cells stimulated by seawater was measured to assess the degree of lung injury. Lung tissues were homogenized in cold phosphate-buffered saline (PBS) (lung tissue to PBS 1:5) and cells were collected and homogenized with repeated freeze-thaw method. Supernatants from tissues and cells were collected by centrifuging at $12,000 \mathrm{rpm}$ for $5 \mathrm{~min}$ at $4^{\circ} \mathrm{C}$. Contents of TNF- $\alpha$ and IL- $1 \beta$ in supernatant were measured according to the manufacturer's instructions.

Evaluation of oxidative stress. T-SOD activity and MDA content were measured to evaluate the status of oxidative stress in lung tissues stimulated by seawater. The lung samples were homogenized in cold PBS (lung tissue to PBS 1:10) and homogenate supernatant was collected by centrifuging at $12,000 \mathrm{rpm}$ for $5 \mathrm{~min}$ at $4^{\circ} \mathrm{C}$. T-SOD activity and content of MDA were measured at 550 and $523 \mathrm{~nm}$, respectively.

Immunohistochemistry study of Trx-1 in lungs. Immunohistochemistry was carried out to evaluate the expression of 
A<smiles>Oc1ccc(/C=C/c2cc(O)cc(O)c2)cc1</smiles>

B<smiles>CC(=O)Oc1ccc(CCc2cc(OC(C)=O)cc(OC(C)=O)c2)cc1</smiles>

Figure 1. The chemical structure of (A) resveratrol (Res) and (B) 3,5,4'-tri-O-acetylresveratrol (AC-Res).

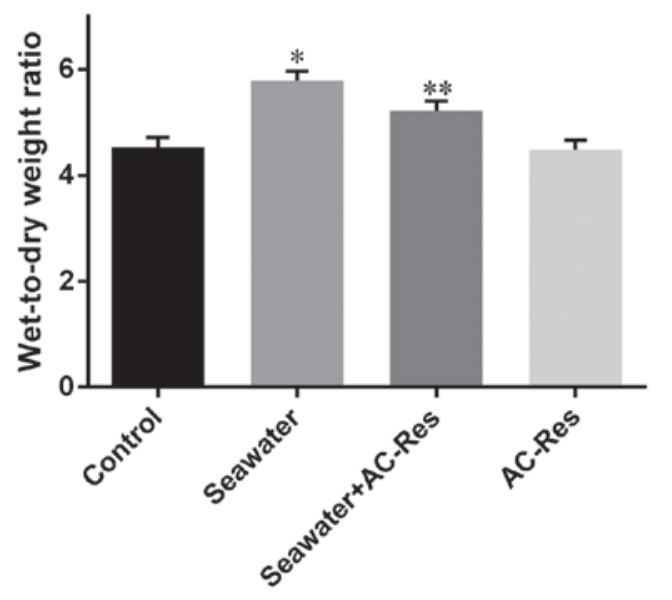

Figure 2. Effects of 3,5,4'-tri-O-acetylresveratrol (AC-Res) on wet to dry ratios (W/D) of lung tissues stimulated with seawater or not. Data are expressed as mean $\pm \mathrm{SD}, \mathrm{n}=8$. Seawater inhalation increased the W/D of lung samples, ${ }^{*} \mathrm{P}<0.0001$ vs. control; while AC-Res pretreatment inhibited the accumulation of water in lungs when stimulated with seawater, ${ }^{* *} \mathrm{P}=0.0002$ vs. seawater inhalation.

Trx-1 in lungs stimulated by seawater or not, as well as the effects of AC-Res on its expression. Briefly, slices of rat lung tissues were deparaffinized, rehydrated in graded alcohol and soaked in $0.3 \% \mathrm{H}_{2} \mathrm{O}_{2}$ at room temperature for $30 \mathrm{~min}$. Slices were blocked with goat serum albumin at $37^{\circ} \mathrm{C}$ for $30 \mathrm{~min}$, and then incubated with the primary antibody against Trx-1 (1:100) at $4{ }^{\circ} \mathrm{C}$ overnight. After that, sections were washed with PBS and incubated in biotin-labeled secondary antibody derived from goat at $37^{\circ} \mathrm{C}$ for $30 \mathrm{~min}$. Then, slices were washed with PBS, incubated in horseradish enzyme-labeled streptavidin working solution and detained with diaminobenzidine (DAB). All slices were checked under a light microscope (DMI6000; Leica, Wetzlar, Germany).

Immunofluorescence detection for Trx-1 in A549 cells. Activity of Trx-1 was detected by immunofluorescence in A549 cells challenged with or without seawater. Briefly, confluent cells grown on coverslips were fixed with methanol at room temperature for $20 \mathrm{~min}$. After being blocked with $0.5 \% \mathrm{BSA}$, cells were incubated with the primary antibody against Trx-1 (1:100) at $37^{\circ} \mathrm{C}$ for $1 \mathrm{~h}$. Then, cells were incubated with CY3 conjugated secondary antibody. Then, nucleus was detained with DAPI at room temperature for $5 \mathrm{~min}$. Finally, cells were examined by using an inverted fluorescence microscope (DMI6000B; Leica).
Western blot analysis. At the end of the experiment, lung and cell samples from different groups were collected and total proteins were extracted according to the manufacturer's instructions (Beyotime Institute of Biotechnology, Jiangsu, China). Equal amount of proteins from each group were separated on sodium dodecyl sulfate-polyacrylamide gel electrophoresis (SDS-PAGE) gel and transferred to PVDF membranes by wet transfer method. Then, membranes were blocked with $5 \%$ non-fat dry milk melted in Tris-buffered saline with $0.1 \%$ Tween-20, and incubated with primary antibodies overnight at $4^{\circ} \mathrm{C}$ against Txnip (1:200), Nrf2 (1:200) and $\beta$-actin (1:2,000). After that, membranes were incubated with secondary anti-body for $2 \mathrm{~h}$ at room temperature followed by 3 times washing. Results of western blot analysis were examined by the enhanced chemiluminescence (ECL) system (Amersham Pharmacia Biotech, Arlington Heights, IL, USA).

Statistical analysis. Statistical analysis was performed with SPSS 17.0 for Windows. Numeric variables were expressed as means \pm SD. Differences between groups were performed by one-way analysis of variance (ANOVA) followed by Dunnett's test. Statistical significance was accepted as $\mathrm{P}<0.05$.

\section{Results}

AC-Res alleviates seawater inhalation-induced lung edema. Water content in lungs from each group was manifested by W/D ratios. Seawater inhalation dramatically increased the $\mathrm{W} / \mathrm{D}$ ratios of lung tissues $(\mathrm{P}<0.05)$, while AC-Res pretreatment significantly inhibited the increasing of water content in lungs manifested by decreased W/D ratios compared with that of seawater inhalation group $(\mathrm{P}<0.05)$ (Fig. 2). On the other hand, administration of AC-Res alone did not affect the content of water in lungs.

AC-Res decreased the content of inflammatory cytokines in lungs. Content of inflammatory cytokines was taken as the symbol of lung injury (Fig. 3) the content of TNF- $\alpha$ (Fig. 3A) and IL-1 $\beta$ (Fig. 3B) significantly increased in lungs stimulated by seawater $(\mathrm{P}<0.05)$. While pretreatment of $A C$-Res inhibited the formation and secretion of TNF- $\alpha$ and IL- $1 \beta$ in lungs stimulated by seawater $(\mathrm{P}<0.05)$. Also, treatment of AC-Res alone did not affect the secretion of cytokines in lung.

Effects of AC-Res on the oxidative stress in lungs stimulated by seawater. In order to evaluate the oxidative stress in lungs 

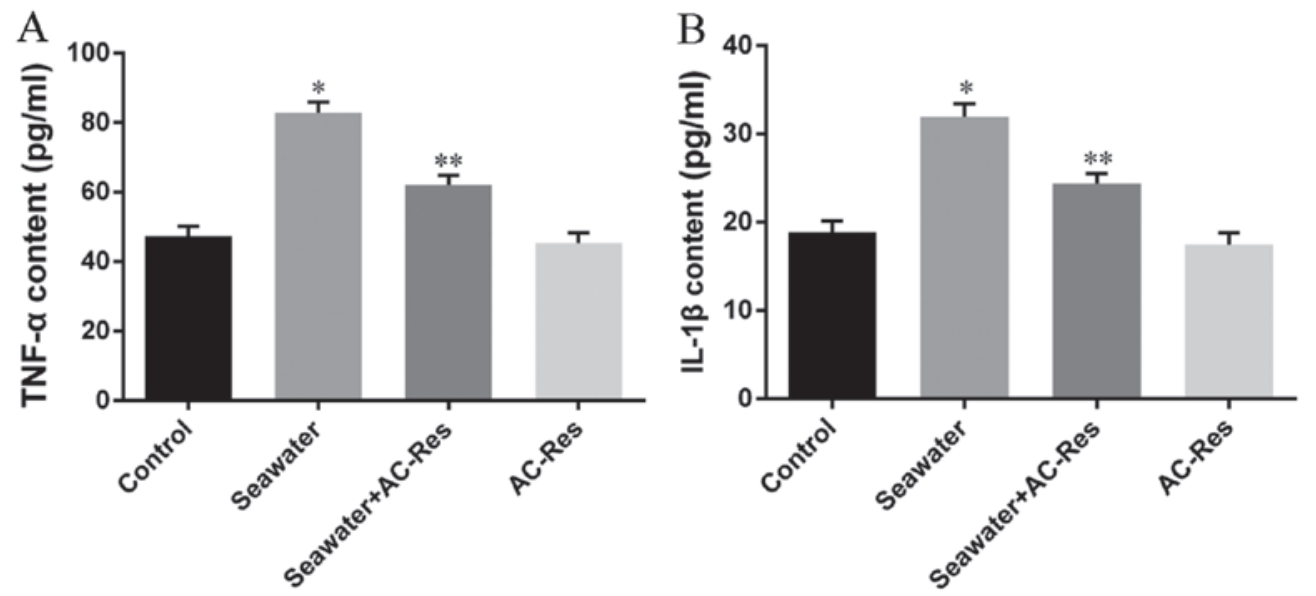

Figure 3. Effects of 3,5,4'-tri-O-acetylresveratrol (AC-Res) on secretion of (A) tumor necrosis factor- $\alpha$ (TNF- $\alpha$ ) and (B) interleukin-1 $\beta$ (IL-1 $\beta$ ) in lungs stimulated by seawater or not. Data are expressed as mean \pm SD, $n=8$. Contents of TNF- $\alpha$ and IL-1 $\beta$ in seawater stimulated lungs were significantly increased, "P $<0.0001$ vs. control; while AC-Res pretreatment decreased the secretion of TNF- $\alpha$ and IL- $1 \beta,{ }^{* *} \mathrm{P}<0.0001$ vs. seawater stimulated lungs.
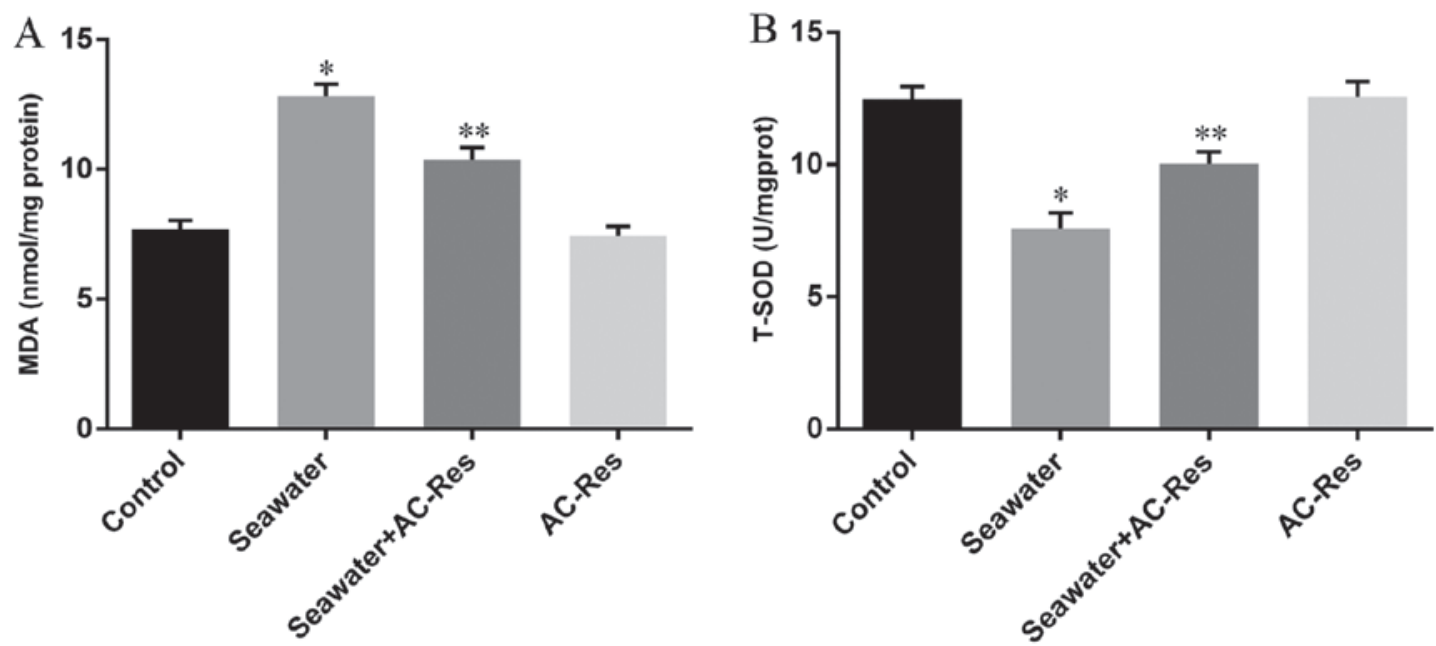

Figure 4. Effects of 3,5,4'-tri-O-acetylresveratrol (AC-Res) on oxidative stress in lungs stimulated by seawater or not. Data are expressed as mean \pm SD, $n=8$. Seawater inhalation increased the content of MDA and decreased the activity T-SOD in lungs, ${ }^{*} \mathrm{P}<0.0001$ vs. control; while AC-Res pretreatment decreased the formation of MDA and upregulated the activity of T-SOD in lungs, ${ }^{* *} \mathrm{P}<0.0001$ vs. seawater inhalation.

challenged by seawater and the protecting effects of AC-Res, The content of MDA and activity of T-SOD were measured by TBA method and hydroxylamine method, respectively. The results (Fig. 4) showed that seawater inhalation led to increased content of MDA (Fig. 4A) together with the decreased activity of T-SOD (Fig. 4B), while pretreatment of AC-Res increased the activity of T-SOD $(\mathrm{P}<0.05)$ and inhibited the formation of MDA $(\mathrm{P}<0.05)$ compared with those in lungs stimulated by seawater. In addition, administration of AC-Res alone did not affect the content of MDA and activity of T-SOD in lungs.

Effects of AC-Res on the expression of Trx-1 axis in lungs stimulated by seawater. In order to explore the mechanisms of oxidative stress in lungs stimulated by seawater and to illustrate how AC-Res inhibited the oxidative stress, we further examined the expression of Trx-1 axis in lungs. As shown in Fig. 5A, immunohistochemistry staining revealed that Trx-1 expressed abundant alveoli epithelium from normal rat lungs, while the expression of Trx-1 dramatically decreased (Fig. 5B) when lungs were challenged by seawater. However, pre-administration of AC-Res strikingly maintained the relative high level expression of Trx-1 in lungs when exposed to seawater (Fig. 5C). Administration of AC-Res alone did not affect the expression of Trx-1 in lungs.

Besides, the expression of key regulators for Trx-1, Nrf2 and Txnip, has also been measured by western blot analysis. As shown in Fig. 6, seawater inhalation upregulated the expression of Txnip (Fig. 6A) and downregulated the expression of Nrf2 (Fig. 6A) $(\mathrm{P}<0.05)$. While pretreatment of AC-Res reversed this trend by inhibiting the express of Txnip increasing Nrf2 expression when lungs were stimulated by seawater $(\mathrm{P}<0.05)$. AC-Res administration alone did not markedly influenced the expression of the two regulators.

Effects of Res on the secretion of cytokines in seawater stimulated NR8383 cells. Based on the findings that seawater inhalation resulted in pulmonary edema and lung inflammation, and pre-administration of AC-Res could alleviate lung injuries by interfering with the Trx-1 axis in lungs. We further investigated the protecting effects of Res, intermediate of 


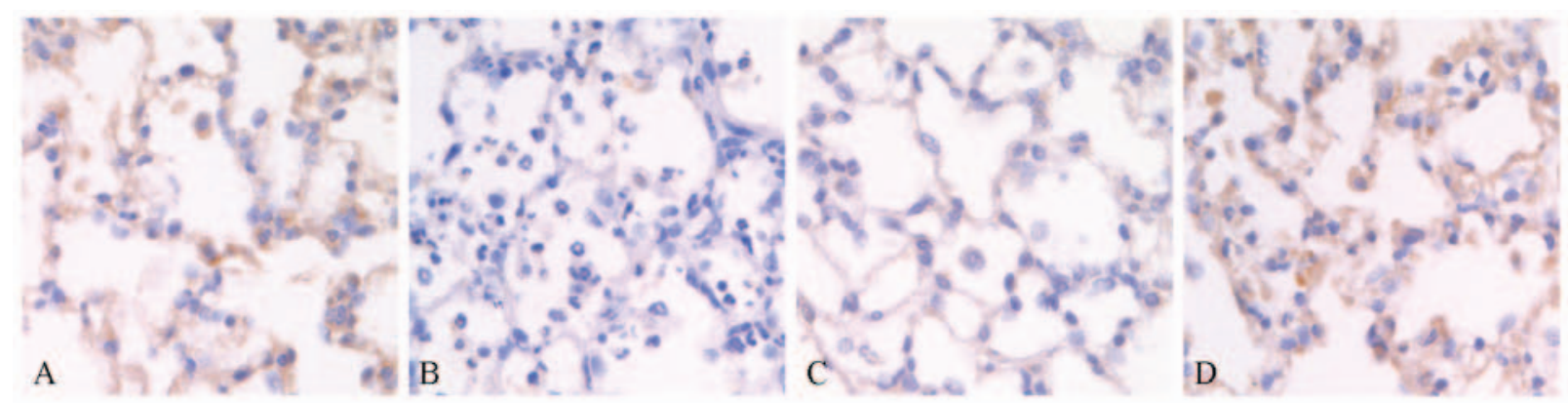

Figure 5. Effects of 3,5,4'-tri-O-acetylresveratrol (AC-Res) on thioredoxin-1 (Trx-1) expression in lungs were tested by immunohistochemistry. (A) Control; (B) seawater inhalation group; (C) seawater inhalation + AC-Res pretreatment group; (D) AC-Res group.
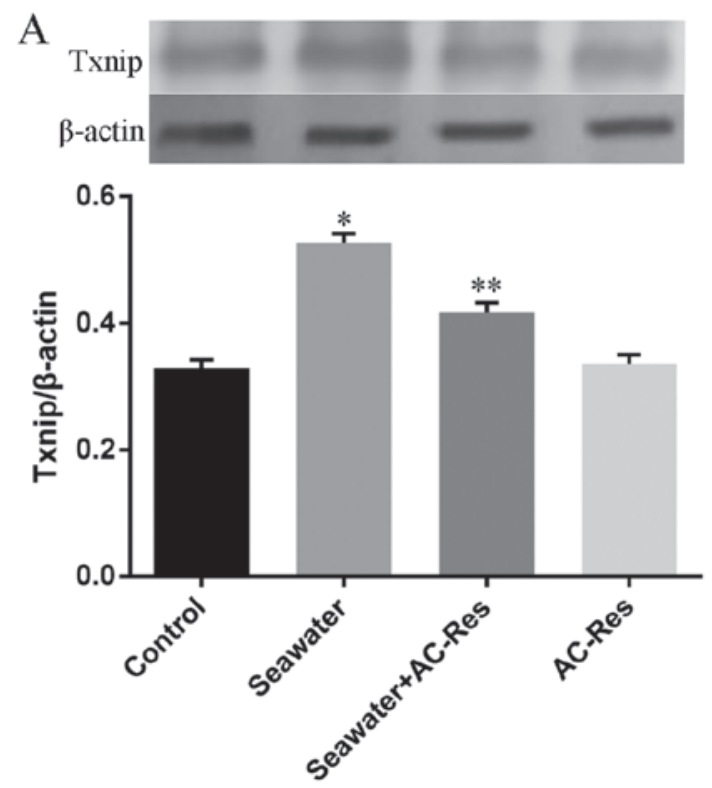
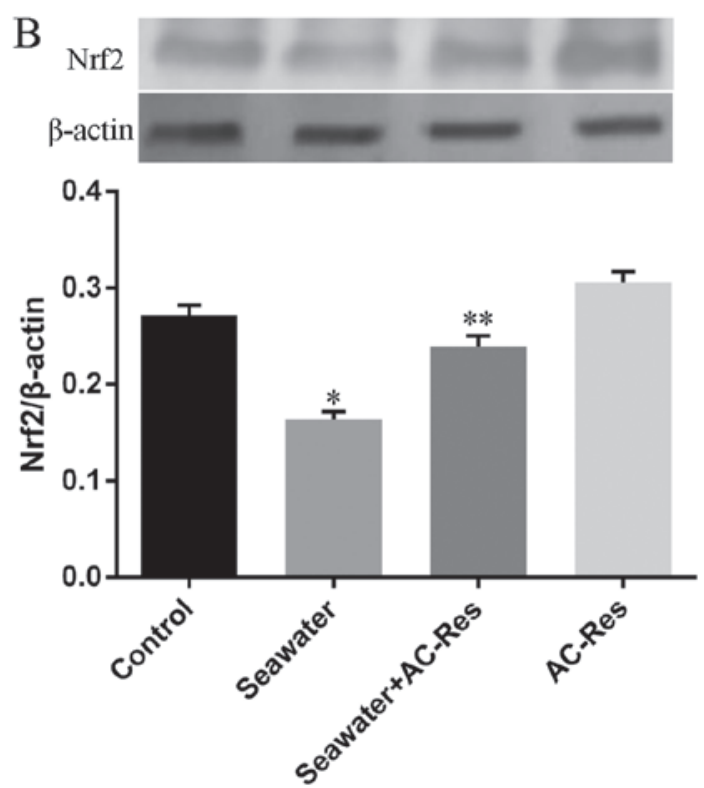

Figure 6. (A and B) Effects of 3,5,4'-tri-O-acetylresveratrol (AC-Res) on the expression of thioredoxin-interacting protein (Txnip) and nuclear factor erythroid 2-related factor 2 (Nrf2) were measured by western blot analysis. Seawater inhalation increased Txnip expression and decreased the expression of Nrf2, ${ }^{*} \mathrm{P}<0.0001$ vs. control; while AC-Res pretreatment decreased the Txnip and increased Nrf2 expression, ${ }^{* *} \mathrm{P}<0.0001$ vs. seawater inhalation.
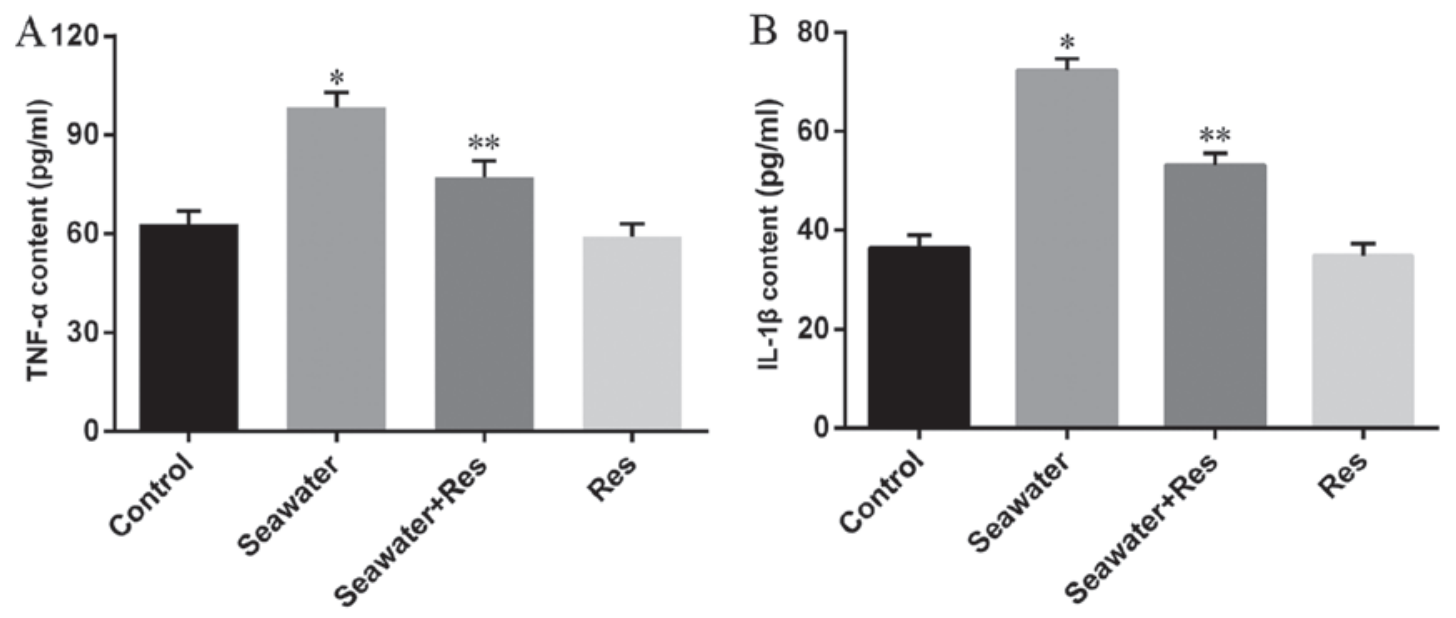

Figure 7. Effects of resveratrol (Res) on formation of (A) tumor necrosis factor- $\alpha$ (TNF- $\alpha$ ) and (B) interleukin-1 $\beta$ (IL-1 $\beta$ ) in NR8383 cells stimulated by seawater or not. Data are expressed as mean $\pm \mathrm{SD}, \mathrm{n}=8$. Seawater stimulation increased the formation of TNF- $\alpha$ and IL-1 $\beta$ in NR8383 cells, ${ }^{*} \mathrm{P}<0.0001 \mathrm{vs}$. control; while Res treatment decreased the secretion of TNF- $\alpha$ and IL- $1 \beta,{ }^{* *} \mathrm{P}<0.0001$ vs. seawater stimulation.

AC-Res, by in vitro experiments. As shown in Fig. 7, formation of TNF- $\alpha$ (Fig. 7A) and IL-1 $\beta$ (Fig. 7B) in NR8383 cells increased when stimulated by $25 \%$ seawater $(\mathrm{P}<0.05)$, while co-incubation of Res decreased the generation of TNF- $\alpha$ and 

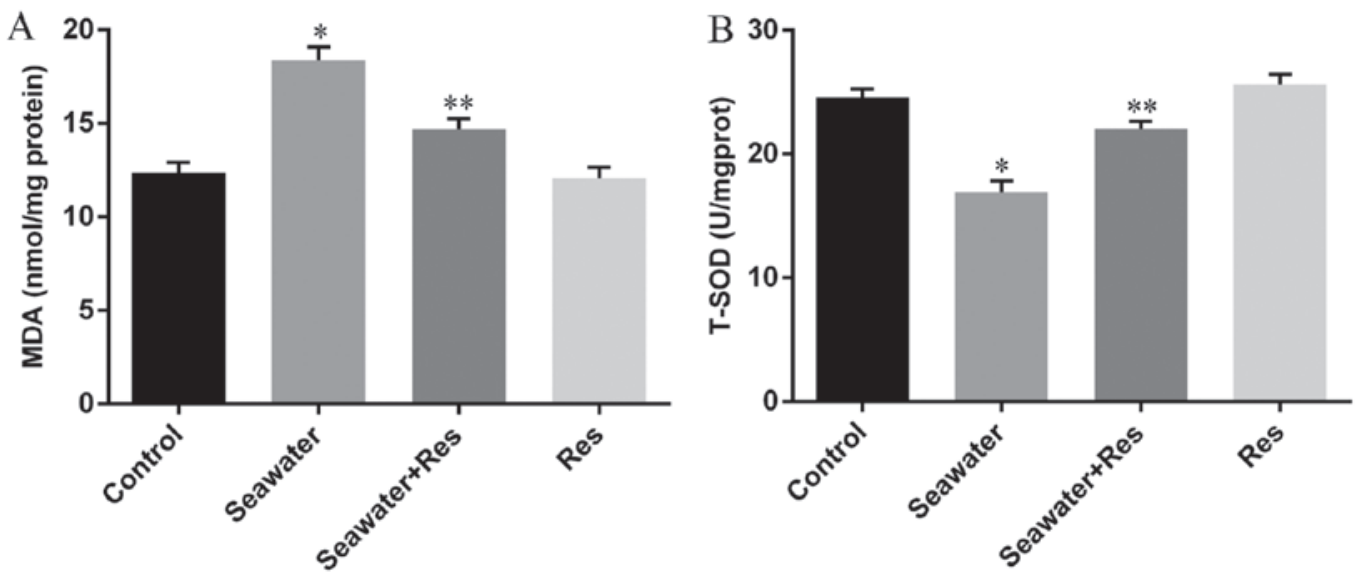

Figure 8. (A and B) Effects of resveratrol (Res) on generation of MDA and activity of T-SOD in NR8383 cells stimulated with seawater or not. Seawater stimulation increased the content of MDA and decreased the activity T-SOD in NR8383 cells, ${ }^{*} \mathrm{P}<0.0001$ vs. control; while Res treatment decreased the MDA content and upregulated the activity of T-SOD in NR8383 cells, ${ }^{* *} \mathrm{P}<0.0001$ vs. seawater stimulation.
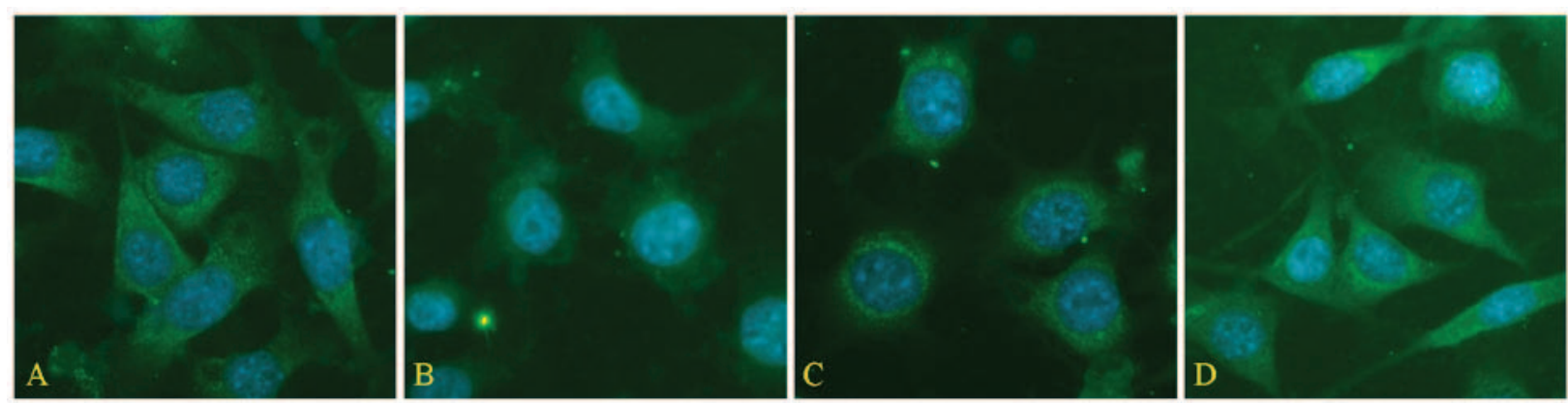

Figure 9. Effects of resveratrol (Res) on thioredoxin-1 (Trx-1) expression in A549 cells were measured by immunofluorescence. (A) Control; (B) seawater exposure group; (C) Seawater exposure + Res pretreatment group; (D) Res group.

IL-1 $\beta(\mathrm{P}<0.05)$ in seawater stimulated cells. In addition, Res treatment alone did not affect the secretion of cytokines in cells.

Effects of Res on the oxidative stress in seawater-stimulated NR8383 cells. Activity of T-SOD and content of MDA were measured to manifest the oxidative stress in NR8383 cells stimulated by seawater. Results (Fig. 8) showed that seawater stimulation increased the content of MDA (Fig. 8A) and inhibited the activity of T-SOD (Fig. 8B) in NR8383 cells $(\mathrm{P}<0.05)$, while Res co-incubation decreased the content of MDA and increased the activity of T-SOD in cells $(\mathrm{P}<0.05)$, which was also similar to the effects of AC-Res on seawater stimulated lungs.

Effects of Res on the expression of Trx-1 axis in seawater stimulated cells. The expression of Trx-1 in seawater-stimulated A549 cells were measured by immunofluorescence detection, and results (Fig. 9B) showed that the expression of Trx-1 decreased in A549 cells $4 \mathrm{~h}$ after seawater exposure while treatment of Res dramatically inhibited the decrease of Trx-1 expression manifested by the relatively higher fluorescence intensity (Fig. 9C).

Also, we detected the expression of Nrf2 and Txnip in seawater-stimulated NR8383 cells. The results showed that seawater stimulation increased the expression of Txnip (Fig. 10A) and decreased Nrf2 expression (Fig. 10B) compared with that of control, while Res co-incubation upregulated the expression of $\mathrm{Nrf} 2(\mathrm{P}<0.05)$ and inhibited the expression of Txnip $(\mathrm{P}<0.05)$ in NR8383 cells when stimulated by seawater.

\section{Discussion}

In the present study, we demonstrated that seawater inhalation resulted in pulmonary edema, inflammation and oxidative stress in lungs, further exploration also revealed that abnormal expression of Trx-1 axis was deeply involved in seawater induced inflammation and oxidative stress imbalance. Based on these findings, we evaluated the effects of AC-Res on seawater-induced oxidative stress and lung injury, and the results showed that AC-Res together with its intermediate, Res, inhibited seawater-induced oxidative stress and lung injuries by interfering the expression of Trx-1 related pathways in vitro and in vivo.

There are basically two different outcomes for drowning victims, one is to die on the drowning spot from suffocation, and the other one is to survive the initial process. However, those who survived the near-drowning process would probably suffer from varied degrees of lung injuries, and maybe ARDS $(4,18)$. Although several pharmacological compounds and therapies have been adopted in clinic for ARDS patients, none have dramatically decreased the motility of ARDS. However, on the bright side the studies have demonstrated that 

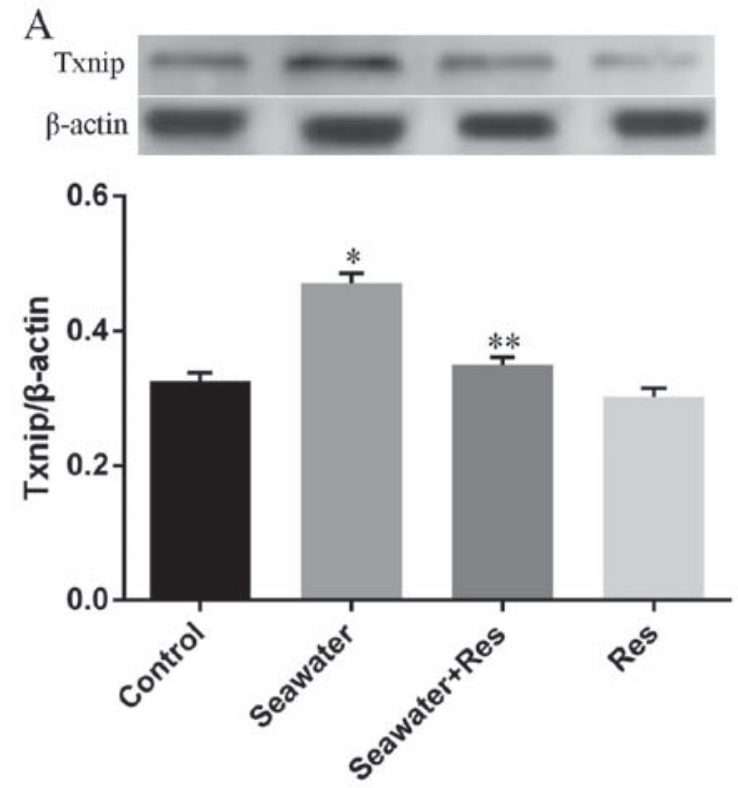

B
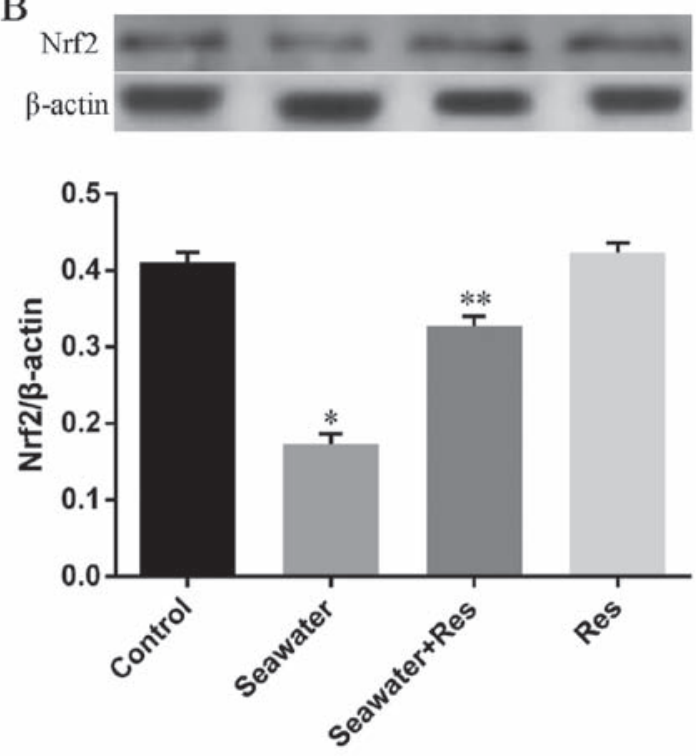

Figure 10. (A and B) Effects of resveratrol (Res) on the expression of thioredoxin-interacting protein (Txnip) and nuclear factor erythroid 2-related factor 2 (Nrf2) in NR8383 cells were measured by western blot analysis. Seawater stimulation increased Txnip expression and decreased the expression of Nrf2 in NR8383 cells, ${ }^{*} \mathrm{P}<0.0001$ vs. control; while Res treatment decreased the Txnip and increased Nrf 2 expression, ${ }^{* *} \mathrm{P}<0.0001$ vs. seawater stimulation.

controlling on inflammation and oxidative stress is beneficial for the outcomes of ARDS patients $(19,20)$.

As known, Res possesses protecting effects on different diseases, such as cardiovascular disorders $(21,22)$, different kinds of cancers $(23,24)$, inflammation $(25,26)$, oxidative stress $(14,27)$ and nervous system disease $(28,29)$. However, Res has never been adopted as a clinical drug due to its poor pharmacokinetic and bio-availability properties $(16,30)$. While as an analog for Res, AC-Res could overcome some of those shortages by extending the biological half-time and inducing the accumulation of Res in lungs (16). Importantly, results from our team and other studies showed that AC-Res possessed anti-inflammation and anti-oxidative stress property which could decrease radiation resulted death and seawater resulted inflammation (31). The present study was designed to further the protecting effects of AC-Res as an antioxidant on seawater induced ARDS.

Seawater inhalation may cause severe pulmonary edema since local high permeability would drive fluid from blood vessels into pulmonary alveoli and lung tissue spaces. Furthermore, neutrophils and macrophages concentrate in lung tissue and secret pro-inflammatory factors, such as NO, COX-2, TNF- $\alpha$, IL-1 $\beta$ and IL-6 (5). Besides, accumulation of inflammatory cells in lung tissues leads to inflammatory responses and oxidative stress imbalance (32). Excessive cytokines and ROS have been recognized to be closely related with the occurrence of ARDS (33). In the present study, it was found that seawater inhalation increased water contents in lungs, enhanced the secretion of TNF- $\alpha$ and IL-1 $\beta$ and inhibited the antioxidant ability of lung tissues. While pretreatment of AC-Res inhibited the infiltration of water from blood vessels into alveolar, decreased the secretion of inflammatory cytokines and rebuilt the antioxidant ability of lung tissues.

We further explored the mechanisms underlying the protecting effects of AC-Res on seawater inhalation induced lung injury and oxidative stress. It is known that the generation of ROS is counterbalanced by antioxidant systems and there are two ROS-scavenging systems in the body: glutathione (GSH) and thioredoxin (Trx) system. As one of the crucial members in the Trx-1 systems, Trx- 1 is a $12 \mathrm{kDa}$ protein which provides electrons to a large range of enzymes and plays a major role in keeping the intracellular redox balance. Trx-1 exhibits protective effects against oxidative stress by scavenging ROS and cooperating with peroxiredoxin (Prdx) (33). In the present study we have found that seawater stimulation inhibited the expression of Trx-1 followed by deregulated T-SOD activity and increased MDA content, while pretreatment of AC-Res in vivo and co-incubation of Res in vitro annihilated the inhibition effects of seawater on activities of Trx-1 followed by upregulated T-SOD activity and decreased MDA content in lungs and cells, respectively.

Besides, it is well known that superabundant of ROS evokes a series of antioxidant genes by activating Nrf2, including the expression of Trx-1 (34). Based on those knowledge, we checked the expression of Nrf2 in seawater stimulated lungs and cells, and the results revealed that seawater exposure inhibited the expression of Nrf2 both in vivo and in vitro, while AC-Res and Res treatment annihilated the effects of seawater and maintained the expression of $\mathrm{Nrf} 2$ at a relative high level. Besides, Txnip has been confirmed to combine with Trx-1 and suppress Trx-1 mediated pro-survival signaling and antioxidant process $(11,35)$. Therefore, we evaluated the activity of this negative regulator in the present study, and results show that seawater stimulation enhanced the expression of Txnip in lungs and cells, while AC-Res and Res treatment inhibited the effects of seawater on Txnip expression both in vitro and in vivo.

In conclusion, the results from the present study revealed that abnormal expression of $\operatorname{Trx}-1$ pathway is blamed for the ARDS induced by seawater inhalation. Besides, it was demonstrated that AC-Res pretreatment in vivo and Res co-incubation 
in vitro inhibited pulmonary inflammation and oxidative stress by interfering with the expression of the Trx-1 axis in lung and cell lines stimulated by seawater. Those results provide scientific evidence for AC-Res as the potential agent for seawater inhalation induced ARDS although further investigations are needed in the clinic.

\section{Acknowledgements}

This study was supported by grants from the Military Key Projects in the 12th Five-year Plan of China (project no. CWS13J043).

\section{Competing interests}

The authors declare that they have no competing interests.

\section{References}

1. Koh Y: Update in acute respiratory distress syndrome. J Intensive Care 2: 2, 2014.

2. Organisation, World Health. Disease and injury regional mortality estimates for 2000-2011: Global summary estimates. 2015-1-20, 2013.

3. Simcock AD: Treatment of near drowning - a review of 130 cases. Anaesthesia 41: 643-648, 1986.

4. Gregorakos L, Markou N, Psalida V,Kanakaki M, Alexopoulou A, Sotiriou E, Damianos A and Myrianthefs P: Near-drowning: Clinical course of lung injury in adults. Lung 187: 93-97, 2009.

5. Zhang Y, Zhang B, Xu DQ, Li WP, Xu M, Li JH, Xie XY, Fan QX, Liu W, Mu DG, et al: Tanshinone IIA attenuates seawater aspiration-induced lung injury by inhibiting macrophage migration inhibitory factor. Biol Pharm Bull 34: 1052-1057, 2011.

6. Yang T, Zhang A, Honeggar M, Kohan DE, Mizel D, Sanders K, Hoidal JR, Briggs JP and Schnermann JB: Hypertonic induction of COX-2 in collecting duct cells by reactive oxygen species of mitochondrial origin. J Biol Chem 280: 34966-34973, 2005.

7. Yu HL, Zhao TF, Wu H, Pan YZ, Zhang Q, Wang KL, Zhang CC and Jin YP: Pinellia ternata lectin exerts a pro-inflammatory effect on macrophages by inducing the release of proinflammatory cytokines, the activation of the nuclear factor- $\kappa \mathrm{B}$ signaling pathway and the overproduction of reactive oxygen species. Int J Mol Med 36: 1127-1135, 2015.

8. Lortz S, Gurgul-Convey E, Lenzen S and Tiedge M: Importance of mitochondrial superoxide dismutase expression in insulinproducing cells for the toxicity of reactive oxygen species and proinflammatory cytokines. Diabetologia 48: 1541-1548, 2005.

9. Liu Y, Qiu J, Wang Z, You W, Wu L, Ji C and Chen G: Dimethylfumarate alleviates early brain injury and secondary cognitive deficits after experimental subarachnoid hemorrhage via activation of Keap1-Nrf2-ARE system. J Neurosurg 123: 915-923, 2015.

10. Niso-Santano M, González-Polo RA, Bravo-San Pedro JM, Gómez-Sánchez R, Lastres-Becker I, Ortiz-Ortiz MA, Soler G, Morán JM, Cuadrado A and Fuentes JM; Centro de Investigación Biomédica en Red sobre Enfermedades Neurodegenerativas (CIBERNED): Activation of apoptosis signal-regulating kinase 1 is a key factor in paraquat-induced cell death: Modulation by the Nrf2/Trx axis. Free Radic Biol Med 48: 1370-1381, 2010.

11. Ogata FT, Batista WL, Sartori A, Gesteira TF, Masutani H, Arai RJ, Yodoi J, Stern A and Monteiro HP: Nitrosative/oxidative stress conditions regulate thioredoxin-interacting protein (TXNIP) expression and thioredoxin-1 (TRX-1) nuclear localization. PLoS One 8: e84588, 2013.

12. Zhang C, Li Q, Kang L, Lei X, Zhai X, Zhao S, Zhang C and Dong W: Resveratrol inhibits hyperxia-induced cell apoptosis through up-regulating SIRT1 expression in HPAECs. Xi Bao Yu Fen Zi Mian Yi Xue Za Zhi 31: 590-595, 2015 (In Chinese).

13. Kuno A, Tanno M and Horio Y: The effects of resveratrol and SIRT1 activation on dystrophic cardiomyopathy. Ann NY Acad Sci 1348: 46-54, 2015.

14. Tvrda E, Kovacik A, Tusimova E, Massanyi P and Lukac N: Resveratrol offers protection to oxidative stress induced by ferrous ascorbate in bovine spermatozoa. J Environ Sci Health A Tox Hazard Subst Environ Eng 50: 1440-1451, 2015.
15. Walle T, Hsieh F, DeLegge MH, Oatis JE Jr and Walle UK: High absorption but very low bioavailability of oral resveratrol in humans. Drug Metab Dispos 32: 1377-1382, 2004.

16. Liang L, Liu X, Wang Q, Cheng S, Zhang S and Zhang M: Pharmacokinetics, tissue distribution and excretion study of resveratrol and its prodrug 3,5,4'-tri-O-acetylresveratrol in rats. Phy tomedicine 20: 558-563, 2013.

17. Ma L, Zhao Y, Li B, Wang Q, Liu X, Chen X, Nan Y, Liang L, Chang R, Liang L, et al: 3,5,4'-Tri-O-acetylresveratrol attenuates seawater aspiration-induced lung injury by inhibiting activation of nuclear factor-kappa B and hypoxia-inducible factor- $1 \alpha$. Respir Physiol Neurobiol 185: 608-614, 2013.

18. Ibsen LM and Koch T: Submersion and asphyxial injury. Crit Care Med 30 (Suppl 11): S402-S408, 2002.

19. Janz DR and Ware LB: Biomarkers of ALI/ARDS: Pathogenesis, discovery, and relevance to clinical trials. Semin Respir Crit Care Med 34: 537-548, 2013.

20. Ware LB: Autopsy in ARDS: Insights into natural history. Lancet Respir Med 1: 352-354, 2013.

21. Tang PC, Ng YF, Ho S, Gyda M and Chan SW: Resveratrol and cardiovascular health - promising therapeutic or hopeless illusion? Pharmacol Res 90: 88-115, 2014.

22. Fu DG: Regulation of redox signalling and autophagy during cardiovascular diseases-role of resveratrol. Eur Rev Med Pharmacol Sci 19: 1530-1536, 2015.

23. Ma L, Li W, Wang R, Nan Y, Wang Q, Liu W and Jin F: Resveratrol enhanced anticancer effects of cisplatin on non-small cell lung cancer cell lines by inducing mitochondrial dysfunction and cell apoptosis. Int J Oncol 47: 1460-1468, 2015.

24. Empl MT, Albers M, Wang S and Steinberg P: The resveratrol tetramer r-viniferin Induces a cell cycle arrest followed by apoptosis in the prostate cancer cell line LNCaP. Phytother Res 29: 1640-1645, 2015.

25. Kjær TN, Thorsen K, Jessen N, Stenderup K and Pedersen SB: Resveratrol ameliorates imiquimod-induced psoriasis-like skin inflammation in mice. PLoS One 10: e0126599, 2015.

26. Kimbrough CW, Lakshmanan J, Matheson PJ, Woeste M, Gentile A, Benns MV, Zhang B, Smith JW and Harbrecht BG: Resveratrol decreases nitric oxide production by hepatocytes during inflammation. Surgery 158: 1095-1101, 2015.

27. Guo S, Yao Q, Ke Z, Chen H, Wu J and Liu C: Resveratrol attenuates high glucose-induced oxidative stress and cardiomyocyte apoptosis through AMPK. Mol Cell Endocrinol 412: 85-94, 2015.

28. Ates O, Cayli SR, Yucel N, Altinoz E, Kocak A, Durak MA, Turkoz Y and Yologlu S: Central nervous system protection by resveratrol in streptozotocin-induced diabetic rats. J Clin Neurosci 14: 256-260, 2007.

29. Venturini CD, Merlo S, Souto AA, Fernandes MC, Gomez R and Rhoden CR: Resveratrol and red wine function as antioxidants in the nervous system without cellular proliferative effects during experimental diabetes. Oxid Med Cell Longev 3: 434-441, 2010.

30. Chen X, He H, Wang G, Yang B, Ren W, Ma L and Yu Q: Stereospecific determination of cis- and trans-resveratrol in rat plasma by HPLC: Application to pharmacokinetic studies. Biomed Chromatogr 21: 257-265, 2007.

31. Koide K, Osman S, Garner AL, Song F, Dixon T, Greenberger JS and Epperly MW: The use of 3,5,4'-tri-O-acetylresveratrol as a potential pro-drug for resveratrol protects mice from $\gamma$-irradiation-induced death. ACS Med Chem Lett 2: 270-274, 2011.

32. Forsgren P, Modig J, Gerdin B, Axelsson B and Dahlbäck M: Intrapulmonary deposition of aerosolized Evans blue dye and liposomes in an experimental porcine model of early ARDS. Ups J Med Sci 95: 117-136, 1990.

33. Liu H, Zhang D, Zhao B and Zhao J: Superoxide anion, the main species of ROS in the development of ARDS induced by oleic acid. Free Radic Res 38: 1281-1287, 2004.

34. Kovac S, Angelova PR, Holmström KM, Zhang Y, Dinkova-Kostova AT and Abramov AY: Nrf2 regulates ROS production by mitochondria and NADPH oxidase. Biochim Biophys Acta 1850: 794-801, 2015.

35. Yu M, Geiger B, Deeb $N$ and Rothschild MF: Investigation of TXNIP (thioredoxin-interacting protein) and TRX (thioredoxin) genes for growth-related traits in pigs. Mamm Genome 18: 197-209, 2007. 